\title{
1. An uphill struggle
}

\subsection{KING OF THE HILL . . . FOR A DAY}

Our story starts with snow, and lots of it. In fact, there was nothing really special about it when it fell during winter 2013 because it was exactly the same kind of snow that falls every year in Europe during winter. However, for Netherlands Railways (NS), it was disastrous. NS had just proudly introduced its brand-new Fyra high-speed train sets for passenger service, and the snow brutally exposed the train's many weaknesses. It collected in the air vents, tore off the steel casing that was supposed to protect the equipment under the carriages, and played havoc with the electronics. However, the trains would have failed even without snow. Earlier, when the weather was still fair, roof plates had come off during testing, as had one of the access doors. But things were also wrong inside the train. The inner doors separating the compartments did not always open when required, and some lavatories were installed incorrectly. When trains were stowed at the railway yard during the night, batteries underneath the carriages had caught fire. Come spring 2013, NS was forced to admit that it couldn't get the trains back into shape. It appeared that there were too many design and construction flaws. The train's constructor, Italian rolling stock manufacturer AnsaldoBreda, had been offered many opportunities to fix the flaws but never really delivered satisfactorily. Consequently, and years after the original deadline to deliver operational train sets, the contract with AnsaldoBreda was formally terminated in August 2013. This put NS in a situation where it had to run a high-speed railway concession costing about 100 million euros per year with neither the proper trains to do it nor the time to fix the problems. In the end, the Dutch government had to step in to rescue NS from going under completely. This created real financial troubles for both NS and the Ministry of Infrastructure and caused distrust among passengers who were left in the cold.

The Fyra train sets were to be the concluding piece of an ambitious project to build a high-speed rail connection between Brussels and Amsterdam. The Netherlands has a relatively solid reputation when it comes to planning and implementing complex projects such as this one. So how exactly did this problematic situation come about? We need to look 
into the past for answers. Following the first successes of the Japanese in the 1960s, and later the French and Germans, the Dutch government decided to jump on the bandwagon in the late 1970s and to build its own network of high-speed railways. It was obvious that this was going to cost a great deal of money. One way of dealing with these costs was by deploying financial schemes that were novel to the Dutch situation. An important decision was to tender the concession to operate the network instead of granting it directly to the incumbent operator, NS, as was done traditionally. This decision put NS into a new situation where, for the first time in its history, it had to compete with other market parties for the right to operate train services.

The tender, which the Ministry expected to grant for approximately 100 million euros per year, attracted interest from other operators such as Deutsche Bahn from Germany, Stagecoach from the United Kingdom, and SJ International from Sweden. Under pressure from the Parliament and from the then-popular sentiment that railways in the Netherlands should never be operated by a foreign company, the Minister allowed NS to hand in its bid before the auction. This gave NS the opportunity to grab the concession before its rivals could outbid it. However, the preliminary bid submitted by NS was considerably lower than what the Ministry had in mind. More than just a little annoyed, and publicly scolding NS for being 'arrogant', the Minister rejected the offer and started the auction in earnest.

Now what? NS was suddenly under intense pressure to win the concession and understood that it was not going to win the Minister's sympathy just by being the sole Dutch operator on the playing field. Close to the auction's deadline, and in a bit of a panic, it submitted a new and substantially higher offer that would gain the Dutch state 160 million euros per year. In contrast, the competing offers all floated around 100 million euros per year. Acting quickly, the Ministry accepted this unexpectedly high offer and even persuaded NS to settle at 148 million euros because it sensed that the very high offer could spell financial trouble for NS in the long run. But even that lowered price was still almost 50 million euros higher than the Ministry had expected to extract from the concession, which made it look like a good deal for the government.

As for NS, it had now become king of the hill. It had defeated its rivals, complied with the demands of the Minister and finally got the most coveted right to operate the high-speed railway link, which NS deemed very important for its future operations. Now it was time to deliver. With such an expensive concession, it was obvious that the actual revenue service had to be as efficient as possible. NS looked at buying high-speed train sets from established manufacturers, but the price and operational costs of 
such trains were deemed too high and wouldn't allow NS to earn back the price of its concession. This was an opportunity for AnsaldoBreda to offer a new design that, on paper, could deliver a high capacity and short travel times whilst remaining below the set prices of other manufacturers. In fact, NS had not much of a choice after two of the competing manufacturers retracted their offers. This drove NS into the arms of the Italians, who set out to build the ill-fated Fyra train. The construction process took years longer than envisaged, partly because AnsaldoBreda had never designed and built such a train before, and partly because the designs were revised during construction. After extended trials on the Czech Velim test track and on the Dutch network, the Fyra was finally accepted for commercial service on 9 December 2012. The first passengers were received with cake and drinks, and there was much media attention. After 20 years of decision making, designing, constructing and calculating, the Dutch finally got their own high-speed railway service. Then the snow came.

\subsection{SURVIVING IN A DYNAMIC LANDSCAPE}

By some measures, NS had actually been quite successful. After a long struggle in an ambiguous situation, it had outdone its rivals and for a brief moment it was king of the hill. However, the successful end of the struggle also triggered a new situation in which the stakes had changed substantially. The former strategy of promising something better than its rivals was no longer relevant. It now had to deliver on its assertion that it could run a viable service. Its former competitors had left the arena, and new adversaries had emerged. NS no longer had to stay ahead of Deutsche Bahn or other operators, but it had to prove itself to the Ministry and, above all, to its passengers. In short, the execution of the concession meant a reset of the actors involved, their relative positions and what they aimed to achieve.

We can use the analogy of hill-climbing in a mountainous landscape to get a better understanding of the decision-making and interaction process that led to the rise and fall of NS in this particular case. NS and other train operating companies competed for the optimal outcome, namely getting the concession. To them, the highest peak constituted winning the concession, so they set out to find the best route to reach that proverbial summit. Similarly, the Ministry had to make moves in order to reach its own particular peak, that is, get the highest return for the concession so that it could recoup some of the enormous construction costs.

This mountainous landscape turned out to be quite dynamic. Once NS had reached the peak of winning the concession, the landscape changed with the introduction of different aims, stakes, actors and conditions. This 
metaphorical hill-climb was done; a new one presented itself in the shape of running the concession successfully. To NS, it meant that it had to work hard to reach a new optimum or peak in the changed landscape, that is, to turn the concession into a success. The very same strategy that had made it king of the hill now turned into a liability, as NS was unable to develop a revenue service with which it could fulfil its annual payment to the state whilst delivering reliable services to its passengers.

Students of human behaviour will not be surprised by this. Most of the time, people make decisions that they believe will give them a clear return in the foreseeable future. But exactly how those decisions pan out in the long run is usually obscured by the fog of the future. In addition, the positions of actors in the landscape are mutually dependent; that is, one's own position is conditional on where others are positioned. If those others move, the landscape may move too. In the face of such dynamics, myopic decisions are inherent to human nature. Consider how NS was fully focused on winning the tender because of the pressure exerted on it by others, and only considered the issue of buying the right trains after it had won the concession. In terms of the hill-climbing analogy, it means that the actors try to estimate where the highest peak in the current landscape is positioned so that they can determine how to get there. But what may constitute a high peak in the short run may turn out to be minor peak in a landscape with other higher peaks in the long run, something which could not be seen because people have difficulties predicting the future. As time moves on, the actors struggle to determine which peak they will have to climb using certain routes, understanding that the landscape will shift over time and that peaks will change as a consequence of those shifts. Climbing mountains therefore constitutes an adaptive walk, a changing route through a changing landscape in an attempt to gain the best position relative to others.

Let us also consider the daily experience of the people working for organizations like NS or the Ministry in such a landscape. From start to finish, the whole project had lasted for over 25 years. Very few people were involved continuously during this long period. Throughout these years, the project saw a succession of no fewer than ten Ministers of Transport. Some stayed in office for two full terms; some were forced to step down after less than one year. From their perspective, the project was a lumbering behemoth, a moving train - if you'll excuse the bad pun - they could ride but not really steer. They had to deal with the situation they found upon entering office and left a somewhat altered situation to their successors. The process will have appeared as a slowly unfolding one, with no apparent end state in sight until that end presented itself in a rather undesired fashion. They will have experienced the pressure from their environment to move 
in one direction or another. They will have seen opportunities and threats, and unexpected dead ends. They will have banked on other actors staying put where they didn't, or the other way around: hoped that actors would move where they decided not to. There was probably a sense of direction towards resolution, but such a resolution could also move further into the future as new obstacles emerged and new quick fixes had to be devised. A solution devised for a problem today could be regarded as an obstacle later on. As the process plodded on, a clear end state seemed evasive.

\subsection{ON EVOLUTION AND COLLECTIVE DECISION MAKING}

The hill-climbing analogy used here is just that: an analogy without much in terms of explanation. It does offer narrative power, because it can convey the complexity of actors trying to align or divert in changing circumstances in an attempt to reach their goals in a fairly accessible way. It is not hard to imagine the actors in the Dutch high-speed railway case as short-sighted mountaineers who struggle to improve their situation while the landscape evolves slowly too. The analogy also invites all sorts of complementary narratives about the interactions between actors, for instance that there may be different routes to the same peak, or that cooperation can help actors to reach their peak more quickly, or the reverse: that walking alone will provide a shorter route to a higher peak. Informative though it is, it doesn't render much explanatory power. We would therefore like to move beyond mere analogies. Anybody trying to understand a process of collective decision making where winners can be losers, and that features an overall outcome that no one had wished for, will be hard pressed to find a fitting framework with which to render explanatory power for such a complex puzzle.

Intuitively, the process can be understood as an evolutionary process. There is a relationship between the considerations and actions undertaken by the individual actors and the overall progression through time. Most, if not all, actors will also have experienced a disconnection between what they did individually at a given time and place and the long-term development of the project. Many of the dynamics of the project stretched beyond their time horizon and outside of their span of control. Still, things happened, and there was a progression, if not necessarily improvement, through time. There was pressure on the actors to make decisions, and there were multiple possible outcomes at any given point in time, with some more likely than others. Long-term development, punctuated change, and a non-linear relationship between individual actions and the 
dynamics on the population level: this ticks all the boxes of an evolutionary theory. We follow Sanderson's point of view (1990) that many theories in the social sciences bear the hallmarks of evolutionary thought. Indeed, we believe that collective decision-making processes are examples par excellence of evolutionary processes in the social realm. And by using that term - evolution - we point not at a general understanding of social processes as being long-term and unfolding to some distant point in time but rather specifically at the mechanisms that govern the evolution of collective decision-making processes and the understanding that such processes develop because of the selection pressures exerted on it. These properties can be named and can be used to generate explanatory power with regard to the slow unfolding of such processes into an uncertain future.

If collective decision-making processes are to be understood as evolutionary processes, this raises a whole range of intriguing questions. How do such processes unfold exactly? By what mechanisms are they governed? Do these mechanisms contribute to a directional or a functional law, that is, do they have their own futures locked within or not? What exactly is the relationship between the activities of individual actors and the outcome? What is selection pressure and how is it processed? How does one assign fitness to certain outcomes? Approaching collective decision making from an evolutionary angle will give us a better understanding of the kind of conundrums found in many cases, such as the botched Dutch high-speed railway project, if we are able to dissect the evolutionary mechanisms at work. The aim of this book is to present an evolutionary model of collective decision making, rooted in a naturalistic understanding of empirical cases. To this end, we will deploy models and tools from evolutionary theories. Roughly speaking, such theories come in two variants. The first one is very precise in mapping the exact relationships between actions and outcomes, but suffers from being overly mechanistic and from an overreliance on very simplistic and static assumptions about reality for the models to work. The second one leaves much more room for the provisional and contextual nature of such relationships but suffers from an overemphasis on chance and randomness, and requires constant semantic innovation to suggest that the ordinary is extra-ordinary. We would like to mediate between these two extremes and to offer a third way that has the precision of the first variant without its gross simplifications, and that has the attention to the situated nature of decision making of the second without its suggestion that each action or event is unique.

Naturally, and as we will explain in detail in the next chapter, we are neither the first nor the last to be working on these themes. There is already a venerable body of knowledge on social evolution, and our book will not conclude all the debates. On the contrary, we wish to provide more fuel for 
those discussions. Our specific contribution to this body of knowledge is that we will deploy one of the versatile models from evolutionary biology, the fitness landscape model, to analyse collective decision making. In evolutionary biology, fitness landscapes are used to study speciation and adaptation, that is, the emergence of biological diversity out of common descent and the occurrence of differentiation. Speciation is governed by a complexity of factors, including but not limited to the interaction between environment and species, the internal genetic composition of the species, and the adaptive capacity of the species in the face of (slowly) changing circumstances. A fitness landscape model provides, simultaneously, a model, tool, heuristic, visualization and metaphor with which to analyse that complexity. If applied well, fitness landscapes can also function as the proverbial Swiss army knife for dissecting the intertwined aspects of decision making.

The caveat for this application is in 'applied well'. Transferring a model from biology to the social sciences requires more than just a few considerations and steps. The original model must be understood first, and then transformed and operationalized to suit a different topic. It must be matched with a research method that does justice to social complexity. Most importantly, it needs to be put to the test. We will present a fitness landscape model for collective decision making that (1) facilitates a structured and systematic analysis of collective decision-making processes and (2) allows for an accessible visualization of such processes. To us, the visualization component is at least as important as the analytical component in this day and age where science has progressed beyond overly simple narratives of how actors make decisions. There is a need, now more than ever, for a method to represent such complex processes in a comprehensible yet accessible way as audiences grapple with increasingly versatile reports of how and why people engage in collective decision making. Here, visualization offers a new avenue to accessibly present investigations to wider audiences. Fitness landscapes hold much potential for both visualization and the analysis of collective decision making. This book aims to unlock that potential.

\subsection{OVERVIEW OF THE BOOK}

We will develop our argument in a number of steps. It is necessary to have a closer look at the nature of evolutionary theories first, and to assess how such theories can also inform those who would like to study social processes in general, and collective decision making in particular. Also, we will highlight the origin of the fitness landscape model and discuss the ways in 
which the model is used by others and can be used for our specific aims. This is the core theme of Chapter 2. Chapter 3 covers the philosophy of fitness landscape inquiries and presents the ontological and epistemological foundations of our particular approach. We present the actual model and its details in Chapter 4. In Chapter 5, we demonstrate the main principles of our model, both its basics and its dynamics, in a highly detailed narrative about the attempts to build and operate a high-speed railway in the Netherlands - a case we have already introduced earlier in this chapter. A closer look at three specific dynamic mechanisms of the model is given in Chapter 6, where we highlight each mechanism by demonstrating its value in three empirical studies: local communities in the Gotthard region, Switzerland trying to develop a vision for the future of their region; the city of Rotterdam, the Netherlands trying to realize a sports campus in the city; and the Thai government trying to foster economic growth through the development of an airport, a railway link and an urban district in Bangkok. We synthesize the findings from the individual studies into a characterization of the evolutionary nature of collective decision making and present six archetypes of such processes in Chapter 7.

This book is the result of five years of theoretical and empirical research. Among other undertakings, we have carried out an extensive literature research (Gerrits and Marks, 2014a, 2015), developed a model, and carried out five major empirical studies on the basis of written sources and interviews (Gerrits and Marks, 2014b; Gerrits et al., 2015a, 2015b). We didn't want to clutter the main text with too many details about the sources, so a list of all sources as well as the ways in which we processed the data is supplied in the appendices. As we explain in more detail in Chapter 3, we have decided to work with qualitative data. In order to handle the consequent vast amount of data and to be able to render visuals from that pool of information, we developed an application with which one can structure, code, score and visualize case-based data. This tool is available for all readers to experiment with at www.un-code.org.

Now, let's get to work. 